\title{
Forensic Comparison of Condom as an Evidences Tool in Deciphered Sexual Assault Cases
}

\author{
KESHAV S. KAPGATE ${ }^{1}$, CHARANSING B. GHOTI ${ }^{1}$, SWATI V. GAIKAWAD ${ }^{2}$, \\ SHIVANGI S. APTE ${ }^{1}$, R. R.PAWAR ${ }^{2}$ and VIJAY J. THAKARE ${ }^{1}$ \\ ${ }^{1}$ Regional forensic Science Laboratory Nagpur-440012, India \\ ${ }^{2}$ Directorate of Forensic Science Laboratory, Mumbai-400098, India \\ keshwa8@gmail.com
}

Received 13 April 2019 / Accepted 2 June 2019

\begin{abstract}
In comparison to other violent crimes, sexual assaults suffer from very low prosecution and conviction rates especially in the absence of DNA as biological evidence. As a result, the forensic community needs to utilize other forms of physical and trace contact evidence, like condom and lubricant, in order to provide a link between the victim and the assailant. Condoms may offer sexual assailants a simple and relatively effective means by which they may remove and dispose of the biological evidence of their contact with the victim. When there is finding of condom as exhibit in decomposed form and without any lubrication traces at that time comparison condom exhibit along with control sample is difficult task. Here we show the development of a multidisciplinary spectroscopic and thermal comparison approach of the used condom sample as exhibit with different condom samples seized from crime scene. The techniques have complementary features and used in this study e.g., differential scanning calorimetric (DSC), Thermo gravimetric analysis (TGA) and FT-IR characterization techniques and provide complementary information to retrieve a condom brand spectroscopic pattern and thermal characteristics by weight loss along with $\mathrm{Tg}$ of Latex component in each condom sample. Unique spectroscopic profiles would greatly aid in the screening and identification of the condom, thus adding intelligence to the case under investigation.
\end{abstract}

Keywords: Forensic science, FTIR, STA, Condom, Lubricant, Polydimethylsiloxane, Polyethylene glycol

\section{Introduction}

In cases of sexual assault, the transfer of biological fluids and materials between the assailant and victim provides investigators with valuable evidence of physical contact between the victim and suspect. However, with increasing public awareness of the identifying properties that exchanged biological fluids present and their high evidential value, particularly that of semen, sexual assailants may seek to avoid such transfers and render the evidence inaccessible ${ }^{1}$. The use of a condom and/or lubricating substance produces 
its own associated trace evidence, and the detection and identification of such residues can provide valuable intelligence information as well as potential forensic evidence in court ${ }^{2}$. The trace evidence associated with the use of a condom can originate from the condom, additional use of lubricants or both. The range of condom and personal lubricants available world-wide is considerably diverse and the components present in a lubricant may vary significantly between brands and even varieties of a particular brand. In general, condom and personal lubricants may be divided into four types: silicone-based, polyethylene glycol (PEG)-based, water-based and oil-based with the silicone-based lubricants being the most commonly encountered lubricant on condoms condom and personal lubricants may also contain spermicidal agents, flavors, colors and perfumes. In cases of sexual assault, the driving need for a lubricating substance by the assailant can also lead to the possibility of the forensic scientist encountering the use of alternative products, which may be any slippery substance 'at hand', such as food oils, moisturizing products or medicinal creams. Blackledge et al., developed a method to detect the presence of polydimethylsiloxane (PDMS) lubricant and nonoxynol-9 based on Fourier transform infrared spectroscopy Hollenbeck $e t a l^{3}$. utilized liquid chromatography electrospray ionization mass spectrometry (LC-ESI-MS) and matrix assisted laser desorption/ionization Fourier transform mass spectrometry (MALDI-FTMS) to examine evidence in a sexual assault investigation Maynard et al., ${ }^{4}$ developed a protocol for the analysis of condom and personal lubricants based on a series of instrumental techniques including DRIFTS, gas chromatography-mass spectrometry (GC-MS ${ }^{5}$, liquid chromatography-tandem mass-spectrometry (LC-MS/MS) and pyrolysis $\mathrm{GC}-\mathrm{MS}^{6-8}$.

Although these methods provide a significant tool for the detection of lubricants consistent with condom use, the methods require relatively expensive instrumentation. In addition the protocols suggested require the use of multiple techniques and are time consuming. Therefore, there is a need for an inexpensive, simple and robust method for the analysis of condom sample. Furthermore, methods for reducing analytical data into manageable dimensions for discrimination purposes would be of benefit.

\section{Case history}

Here we discuss one evidence of rape or sexual assault in which accused used a condom for rape later after few days police seized a used condom from location of crime and other condom as control from accused and submitted to the FSL to know whether the same condom used by accused or not, but the used condom is in decomposed condition there are very less chances to get biological evidence from condom sample then the physical comparison of condom play vital role in identification and comparison of condom sample.

We have received used condom sample as exhibit from scene of crime and another condom sample ascontrol seized from accused person. Generally, in forensic science laboratories (FSL) we received many different types of rape cases in earlier cases in penetrative rape generally do not involve use of condom. Now a day's people become aware and use a condom during sexual intercourse to reduce probability pregnancy or sexually transmitted disease. The forensic system work to collect, prevent and understand evidences that can help to prove crime, therefore condom is also very important circumstantial evidence in rape case to prove crime. Whether the same condom used by accused when there is no other evidence present. When condom is in used or decomposed condition there is very less chances to get a lubricant from same in that condition material of condom can also a important evidence to prove crime. 
FSL Maharashtra have a highly sophisticated instrument differential scanning calorimetric (DSC), Thermo gravimetric analysis (TGA) and FT-IR. This present study investigated the evidential value by the physical comparison of condom sample.

\section{Experimental}

Here we have condom sample seized from location of crime and control sample of condom collected from accused person. Condom sample were cut into small pieces and 4-5 mgs of sample were encapsulate in aluminum crucible with lid and same used for STA analysis. $5 \mathrm{mg}$ Condom sample is used for FTIR analysis with ATR mode.

\section{Instrumentation}

\section{ATR-Fourier transforms infrared analysis}

FTIR spectra were recorded on Perkin Elmer FTIR spectrometer (Model: Frontier, Serial No. 91579) spectrometer Frontier using ATR transmittances are reported in $\mathrm{cm}^{-1}$. Condom sample were studied using scan with ATR mode in the range of $4000-400 \mathrm{~cm}^{-1}$. Images were processed using FTIR spectrometer Frontier using ATR transmittance and images were recorded at $4 \mathrm{~cm}^{-1}$ spectral resolution averaging 256 scans over a wavenumber $4000-400 \mathrm{~cm}^{-1}$.

\section{Thermal analysis by simultaneous thermal analysis (STA)}

The STA measurements were performed using NETZCSH STA 449 F3 (Jupiter) instruments using aluminum crucible with pierced lid and nitrogen as purge gas at flow rate of $40 \mathrm{~mL}$ /min. About 5-6 mg samples were used in this study. During study, samples were placed in the center of the aluminum crucible with tweezers and each specimen was encapsulate. The analysis was performed in the range of $50{ }^{\circ} \mathrm{C}$ to $600{ }^{\circ} \mathrm{C}$ at the rate of $20 \mathrm{~K} / \mathrm{min}$. Data was analyzed using NETZCSH protease software.

\section{Results and Discussion}

From decomposed condition there are very less chances to get biological evidence from condom sample then the physical comparison of condom play vital role in identification and comparison of condom sample so we have used Physical method for comparison of condom sample STA and FTIR analysis.

\section{IR Spectral characterization}

Characteristic infrared bands in control condom and exhibit can be seen in Figure 1 at 3030 $\mathrm{cm}^{-1}$ (asymmetric $=\mathrm{CH}$ stretch), $2965 \mathrm{~cm}^{-1}$ (asymmetric $\mathrm{CH}_{3}$ stretch), $2923 \mathrm{~cm}^{-1}$ (asymmetric $\mathrm{CH}_{2}$ stretch), $2846 \mathrm{~cm}^{-1}$ (symmetric $\mathrm{CH}_{3}$ stretch), $2719 \mathrm{~cm}^{-1}$ (symmetric $\mathrm{CH}_{2}$ stretch), $1666 \mathrm{~cm}^{-1}$ (C=C stretch), $1478 \mathrm{~cm}^{-1}, 1451 \mathrm{~cm}^{-1}$ and $1378 \mathrm{~cm}^{-1}\left(\mathrm{CH}_{2}, \mathrm{CH}_{3}\right.$ deformation), $1308 \mathrm{~cm}^{-1}\left(\mathrm{CH}_{2}\right.$ rock), $1266 \mathrm{~cm}^{-1}$ (CH deformation), $1128 \mathrm{~cm}^{-1}, 1093 \mathrm{~cm}^{-1}$ and $1047 \mathrm{~cm}^{-1}\left(\mathrm{CH}_{2}\right.$ rock), $932 \mathrm{~cm}^{-1}$ (cis $\mathrm{CH}$ wag vibration), $889 \mathrm{~cm}^{-1}, 843 \mathrm{~cm}^{-1}\left(\mathrm{CH}_{2}\right.$ wag) and $751 \mathrm{~cm}^{-1}$ (cis $\mathrm{CH}$ wag). And functional characteristics band of latex material is observed here in both condom sample.

The characteristics IR band of control condom sample due to asymmetric $=\mathrm{CH}$ stretch, asymmetric $\mathrm{CH}_{3}$ stretch and asymmetric $\mathrm{CH}_{2}$ stretch tallies with exhibit's IR band (Figure 1).

\section{Thermal characteristics of control (Condom) and exhibit sample}

Thermogravimetric analysis (TGA) is one of the members of the family of thermal analysis techniques used to characterize a wide variety of materials.TGA provides complimentary and supplementary characterization information to the most commonly used thermal 
technique, DSC. TGA measures the amount and rate (velocity) of change in the mass of a sample as a function of temperature or time in a controlled atmosphere. The measurements are used primarily to determine the thermal and/or oxidative stabilities of materials as well as their compositional properties. The technique can analyze materials that exhibit either mass loss or gain due to decomposition, oxidation or loss of volatiles (such as moisture). It is especially useful for the study of polymeric materials, including thermoplastics, thermosets, elastomers, composites, films, fibres, coatings and paints ${ }^{9}$.

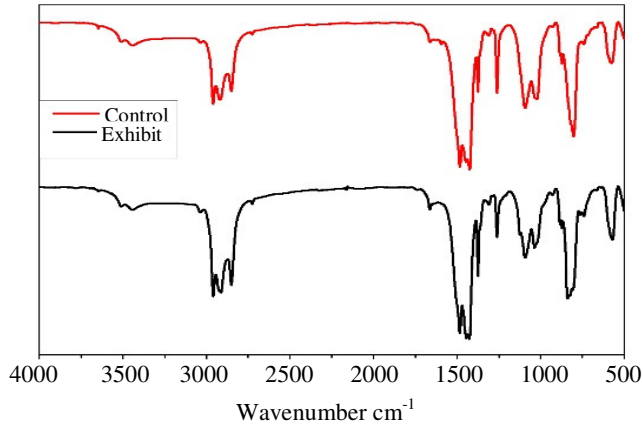

Figure 1. FTIR spectra of condom sample (Control and exhibit)

\section{Thermogravimetry and DTG analysis of control (Condom) and exhibit sample}

Thermal analysis of control condom and exhibit are shown in Figure 2, which indicate decomposition temp of control condom and exhibit in the range of $320-460{ }^{\circ} \mathrm{C}$ with mass change of $96.01-97.10 \%$. Differential thermogravimetric analysis of control condom and exhibit shows shows peak at $389.1{ }^{\circ} \mathrm{C}$ and $391.2{ }^{\circ} \mathrm{C}$. The result of thermal analysis depicted in Figure 2 and Table 1 clearly shows the perfect matching curves of control condom and exhibit.

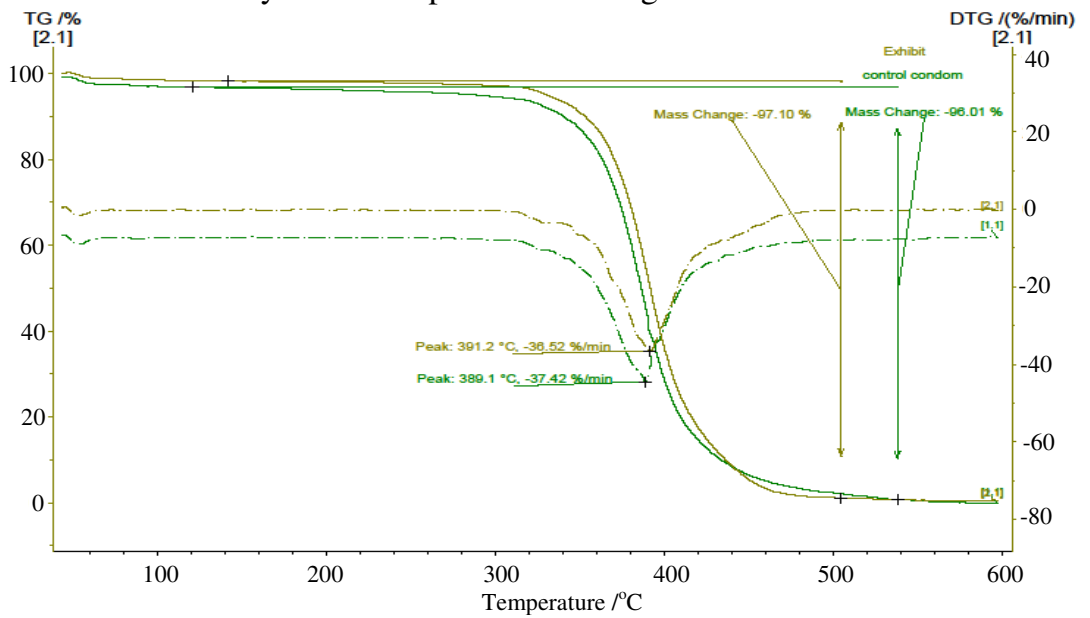

Figure 2. TG and DTG of Condom Sample (Control and exhibit)

\section{Differential scanning colorimetry}

The result of differential scanning colorimetry depicted in Figure 3 and Table 1 clearly shows the perfect matching temperatures of control condom sample and exhibit. The figure shows three DSC curves at temperature range $220.5-225.6{ }^{\circ} \mathrm{C}, 340-340.6{ }^{\circ} \mathrm{C}$ and $325.4-$ $332.9^{\circ} \mathrm{C}$. 


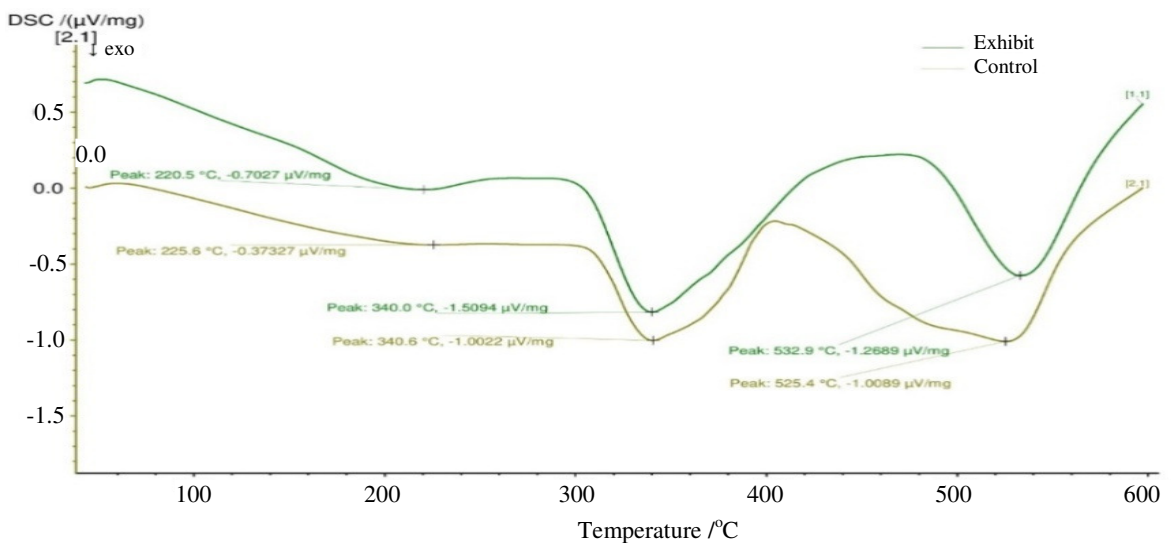

Figure 3. DSC curves of condom sample (Control and exhibit)

Table 1. DSC, TG and DTG result for exhibit and control sample

\begin{tabular}{cccccc}
\hline Sample & \multicolumn{3}{c}{ DSC Curve peak } & Mass change & $\begin{array}{c}\text { DTG Curve } \\
\text { peak }\end{array}$ \\
\hline & Temp.,${ }^{\circ} \mathrm{C}$ & Temp.,${ }^{\circ} \mathrm{C}$ & Temp., ${ }^{\circ} \mathrm{C}$ & & Temp.,${ }^{\circ} \mathrm{C}$ \\
\hline Control & 220.5 & 340 & 532.9 & $96.01 \%$ & 389.1 \\
Exhibit & 225.6 & 340.6 & 525.4 & $97.10 \%$ & 391.2 \\
\hline
\end{tabular}

\section{Conclusion}

The ability to detect a wide polymer as latex associated with a condom suggests the opportunity to discriminate between different condom brands/types thus enhancing the value of the forensic intelligence provided for the sexual assault case being investigated. A multidisciplinary analytical approach serves this purpose by providing complementary information. The DSC, TGA and ATR-FTIR could be used in an integrated work can be protocol such as that suggested by this workwould enable comparison andmatch of crime scene condom with its control by using its thermal behavior and spectral characteristicdatabase to be built using informationsuch as those provided by these investigations, a part of whichhave been reported in the present study.

\section{Acknowledgement}

The Authors wish to thank Dr. K. V. Kulkarni, Director, Directorate of Forensic Science Laboratories, State of Maharashtra, Mumbai, India, for encouraging and supporting us.

\section{References}

1. Blackledge R D, Condom Trace Evidence: A new Factor in Sexual Assault Investigation. FBI Law enforcement Bulletin Volume: 65 Issue: Dated:(May 1996) Pages: 12-16; https://www.ncjrs.gov/App/publications/abstract.aspx?ID=161290

2. Maynard P, Allwell K, Roux C, Dawson M and Royds D, Forens Sci Int., 2001, 124(2-3), 140-156; DOI:10.1016/S0379-0738(01)00588-6

3. Blackledge R D and Vincenti M, J Forens Sci Soc., 1994, 34(4), 245-256;

DOI:10.1016/S0015-7368(94)72928-5

4. Blackledge R D, J Forens Sci., 1995, 40(3), 467-469. 
5. Hollenbeck T, Siuzdak G and Blackledge R, J Foren Sci., 1999, 44, 783-788;

DOI:10.1520/JFS14553J

6. Diamantaras K I and Kung S Y, Principal Component Neural Networks: Theory and Applications, Wiley, New York, NY, 1996.

7. Brown S D, Appl Spectrosc., 1995, 49(12), 14A-31A.

8. Fiona Burger, Michael Dawson, Claude Roux, Philip Maynard, Philip Doble and Paul Kirkbride, Talanta, 2005, 67(2) 368-376; DOI:10.1016/j.talanta.2005.03.040 